\title{
Capacity Analysis of Correlated MIMO Channels
}

Leif Hanlen ${ }^{1}$

School of Elec. Engineering \& Comp. Science Univ. of Newcastle, Australia

e-mail: leifdee.newcastle.edu.au
Alex Grant ${ }^{2}$

Institute for Telecommunications Research

Univ. of South Australia, Adelaide, Australia

e-mail: alex.granteunisa.edu.au
Abstract - The capacity of correlated finite-dimensions MIMO channels, where the channel gains have a generalized Wishart distribution is found. Asymptotic expressions are given where one dimension is much larger than the other. For many transmitters, the asymptotic capacity can be divided into two components: one arising from the dominant eigenvalues of the correlation matrix, and the other from the remaining eigenvalues.

\section{SUMMARY}

The work of [1-3] has shown that under the assumption of an i.i.d. transfer matrix, the capacity of a MIMO channel grows in proportion to the minimum number of transmitters and receivers. Recently, [4] proposed the use of the Stieltjes' transform to estimate the capacity of a correlated channel, and suggested that the growth of the MIMO channel will remain linear under correlation, although the proportionality constant may change.

Consider a point-to-point communication link with $t$ transmit antennas and $r$ receive antennas. Define $m=\min \{r, t\}$ and $n=$ $\max \{r, t\}$. At each symbol interval, $y \in \mathbb{C}^{r}$ depends on $x \in \mathbb{C}^{t}$,

$$
y=H x+w
$$

Element $y_{j}$ is the matched-filter output from antenna $j$, while $x_{i}$ is the signal transmitted from antenna $i$, with the transmitter given a transmission power limit $P$. The matrix $H \in \mathbb{C}^{r \times t}$ has elements $H_{j i}$, which are the complex gains between transmit antenna $i$ and receive antenna $j$. The vector $w \in \mathbb{C}^{r}$ contains i.i.d. circularly symmetric Gaussian noise samples $\mathrm{E}\left[w w^{*}\right]=\eta^{2} I_{r}$. The $H_{j i}$ are chosen from a complex Gaussian ensemble with zero mean and an $m \times m$ covariance matrix $\Sigma$. In the notation of [6], $H \sim N_{r, t}\left(0, \Sigma \otimes I_{n}\right)$. If $\Sigma=I_{m}$ we have the well known i.i.d. case [1].

Assume $H$ is known at the receiver and that $H, \Sigma$ are unknown at the transmitter. In this case, $\mathrm{E}\left[x x^{*}\right]=P \cdot I_{t} / t$ is optimal.

\section{Theorem 1 (Correlated MIMO Capacity).}

The capacity of the ergodic correlated MIMO channel (1) with $H \sim N_{r, t}(0, \Sigma \otimes I)$ is given by

$$
\begin{array}{r}
C=\frac{n^{m n} \pi^{m(m-1)}}{2^{m n} \Gamma_{m}(n) \Gamma_{m}(m) \operatorname{det}(\Sigma)^{n}} \int_{\Lambda}{ }_{0} F_{0}\left(-\frac{1}{2} \Sigma^{-1}, n \Lambda\right) \\
\cdot \prod_{i=1}^{m} \lambda_{i}^{(n-m)} \prod_{i<j}^{m}\left(\lambda_{i}-\lambda_{j}\right)^{2} \sum_{i=1}^{m} \log \left(I+\frac{P}{t} n \lambda_{i}\right) d \Lambda
\end{array}
$$

where $\Gamma_{m}(a)=\pi^{m(m-1) / 2} \prod_{i=1}^{m} \Gamma(a-i+1)$ is the complex multivariate gamma function, $\Lambda=\operatorname{diag}\left(\lambda_{1}, \ldots, \lambda_{m}\right)$ and ${ }_{0} F_{0}(\cdot)$ is a hypergeometric function of two matrix arguments [6, p. 34].

\footnotetext{
${ }^{1}$ A part of this work was carried out while L. Hanlen visited the Institute for Telecommunications Research.

${ }^{2}$ The work of A. Grant was supported in part by the Australian Government under ARC Grant DP0209658.
}

\section{Theorem 2 (Asymptotic Correlated MIMO Capacity).}

Consider (1) with $H \sim N_{r, t}(0, \Sigma \otimes I)$ such that $\Sigma$ has eigenvalues $\sigma_{1}>\cdots>\sigma_{k}>\sigma_{k+1}=\cdots \sigma_{m}=\sigma>0$. [5, Corrollary 9.5.7]. Then the asymptotic capacity $C_{\infty}$, as $n \rightarrow \infty$ with finite $m$ is

$$
\begin{aligned}
& C_{\infty} \sim \sum_{i=1}^{k} \log \left(1+\frac{P}{t} n \sigma_{i}\right) \\
&+\int_{-\infty}^{\infty} \log \left(1+\frac{P}{t} n \sigma_{m}\left[z\left(\frac{n}{2}\right)^{-\frac{1}{2}}+1\right]\right) \\
& \cdot \sum_{j=1}^{m-k} \frac{\left[H_{j}\left(\frac{z}{\sqrt{2}}\right)\right]^{2}}{2^{j} j ! \sqrt{2 \pi}} e^{-z^{2} / 2} d z
\end{aligned}
$$

where $H_{j}(\cdot)$ is the $j$-th Hermite polynomial [7] and $A \sim B$ implies $A / B \rightarrow 1$.

When $r=n \rightarrow \infty$ and $m=t$, capacity grows logarithmically without bound. For $r=m$ and $n=t \rightarrow \infty$, the right hand side above converges to a constant.

Corollary 1 (Large $t$ ).

Suppose $t \gg r$, i.e. $t=n \rightarrow \infty$ and $r=m$, then

$$
\lim _{t=n \rightarrow \infty} C_{\infty} \approx \sum_{i=1}^{k} \log \left(1+P \sigma_{i}\right)+(m-k) \log \left(1+P \sigma_{m}\right)
$$

where we have omitted constants of integration.

Corollary 1 presents two forms of growth for increasing $r$. The first term is given by the dominant eigenvalues of the covariance matrix and for $r \geq k$ is independent of $r$. The second term is linear in $r-k$ and corresponds to "linear" capacity growth of the i.i.d. channel, with proportionality constant $\alpha=\log \left(1+P \sigma_{m}\right)$. Over the class of equivalent covariance matrices $S_{m}=\left\{\Sigma_{m} \in S_{m}: \operatorname{tr}\left(\Sigma_{m}\right)=m\right\}$ the i.i.d. channel has the largest rate of capacity growth $\alpha=\log (1+P)$ for increasing $r$.

\section{REFERENCES}

[1] I. E. Telatar, "Capacity of Multi-antenna Gaussian Channels," European Trans. Telecommun, vol. 10, no. 6, Nov, pp. 585-595, 1999.

[2] G. J. Foschini and M. J. Gans, "On Limits of Wireless Communications in a Fading Environment when using Multiple Antennas," Wireless Personal Communications, vol. 6, pp. 311-335, 1998.

[3] A. J. Grant, "Rayleigh Fading Multiple-antenna Channels," EURASIP Journal on Applied Signal Processing, Special Issue on Space-Time Coding, pt. I, no. 3, Mar, pp. 316 - 329, 2002.

[4] C.-N. Chuah, D. N. C. Tse et.al,"Capacity Scaling in MIMO Systems Under Correlated Fading," IEEE Trans. Inform. Theory, vol. 48, no. 3, Mar, pp. 637-650, 2002.

[5] R. J. Muirhead, Aspects of Multivariate Statistical Theory, John Wiley \& Sons, Inc, New York, 1982.

[6] A. K. Gupta and D. K. Nagar, Matrix Variate Distributions, Chapman \& Hall/CRC, Boca Raton, 2000.

[7] G. Szegö, Orthogonal Polynomials American Mathematical Society, Providence, RI, 1939. 